\title{
Photoionization and transient Wannier-Stark ladder in silicon: First-principles simulations versus Keldysh theory
}

\author{
Thibault J.-Y. Derrien $10,{ }^{1, *}$ Nicolas Tancogne-Dejean ${ }^{\circ},{ }^{2}$ Vladimir P. Zhukov, ${ }^{1,3}$ \\ Heiko Appel, ${ }^{2}$ Angel Rubio, ${ }^{2}$ and Nadezhda M. Bulgakova ${ }^{1, \dagger}{ }^{1, \dagger}$ \\ ${ }^{1}$ HiLASE Centre, Institute of Physics, Academy of Science of the Czech Republic, Za Radnicí 828/5, 25241 Dolní Břežany, Czech Republic \\ ${ }^{2}$ Max Planck Institute for the Structure and Dynamics of Matter (MPSD), Luruper Chaussee 149, 22761 Hamburg, Germany \\ ${ }^{3}$ Federal Research Center for Information and Computational Technologies, 6 Lavrentyev Ave., 630090 Novosibirsk, Russia
}

(Received 20 August 2021; revised 13 November 2021; accepted 16 November 2021; published 22 December 2021)

\begin{abstract}
Nonlinear photoionization of dielectrics and semiconductors is widely treated in the framework of the Keldysh theory whose validity is limited to photon energies that are small compared to the band gap and relatively low laser intensities. The time-dependent density functional theory (TDDFT) simulations, which are free of these limitations, enable one to gain insight into nonequilibrium dynamics of the electronic structure. Here we apply TDDFT to investigate the photoionization of silicon crystal by ultrashort laser pulses in a wide range of laser wavelengths and intensities and compare the results with predictions of the Keldysh theory. Photoionization rates derived from the simulations considerably exceed the data obtained with the Keldysh theory within the validity range of the latter. Possible reasons for the discrepancy are discussed and we provide fundamental data on the photoionization rates beyond the limits of the Keldysh theory. By investigating the features of the Stark shift as a function of photon energy and laser field strength, a manifestation of the transient Wannier-Stark ladder states is revealed, which become blurred with increasing laser field strength. Finally, it is shown that the TDDFT simulations can potentially provide reliable data on the electron damping time that is of high importance for large-scale modeling.
\end{abstract}

DOI: 10.1103/PhysRevB.104.L241201

The Keldysh photoionization theory for atoms and solids published in 1964 [1] plays a vital role in interpretation of ultrafast laser-induced phenomena, the field which continues to rapidly develop and influence our knowledge about fundamental processes in physics, chemistry, and biology. However, its applicability is limited by the requirements of a sufficiently small photon energy compared to ionization potential (band gap in solids) [1,2] and not too strong laser fields [3]. For band-gap solids, the theory [1] enables calculations of the number of electrons excited from the valence to the conduction bands per time unit, using a simplified description of the electronic levels reduced to two bands. This simplification has become commonly employed for qualitative simulations of laser-induced materials damage [4-6] while experimental observations indicate that the Keldysh theory can considerably underestimate or overestimate photoionization rates, depending on irradiation regime and kind of solid $[2,7,8]$. Direct experimental investigations of multiphoton inner excitation of band-gap materials was made possible relatively recently [9-12]. Supported by comparisons with experimental

\footnotetext{
*derrien@fzu.cz

†bulgakova@fzu.cz
}

Published by the American Physical Society under the terms of the Creative Commons Attribution 4.0 International license. Further distribution of this work must maintain attribution to the author(s) and the published article's title, journal citation, and DOI. data, several modifications of the Keldysh model for solids were proposed to address discrepancies between experiment and theory $[3,8,13,14]$.

An important feature of the original Keldysh theory is the dependence of the effective ionization potential for atoms and the effective band gap energy in the case of crystals, $U_{\text {eff }}$, on the laser field intensity [1]. Although the increase of the $U_{\text {eff }}$ value with intensity was proven experimentally for atomic gases $[15,16]$, an eventual increase of the band gap energy in crystals $[4,17]$ irradiated by linearly polarized light was, to our knowledge, not observed for bulk materials. Recently, a clear shift of the energy levels to higher values was found in monolayer $\mathrm{WS}_{2}$ [18] under irradiation by circularly polarized femtosecond laser pulses while a replication of the electronic levels by laser dressing was well demonstrated in GaAs [19]. Moreover, recent experimental studies performed for $\mathrm{Si}$ and $\mathrm{ZnO}[20,21]$ supported a reduction of the band gap energy in the laser field. These findings are consistent with the Keldysh theory where, in the multiphotonic regime, $U_{\text {eff }}$ increases with the field amplitude $(\gamma \gg 1$ with $\gamma$ to be the Keldysh adiabadicity parameter) whereas in the tunneling regime the contribution of $U_{\text {eff }}$ fully fades out $(\gamma \ll 1$ ). Nevertheless, the interpretations of $\gamma$ and $U_{\text {eff }}$ remain elusive, particularly for the cases of the mixed regime $(\gamma \sim 1)$.

Keldysh [1] attributed the increase of $U_{\text {eff }}$ to the Stark effect. As a whole, the Stark shift can transiently affect the band gap energy at high intensities while the laser dressing may eventually close the band gap, e.g., in the regime when $\gamma \ll 1$ [22]. However, there is no clarity of the manifestation 
and interplay of these effects at different irradiation regimes that is of high importance for predicting the consequences of the action of ultrashort laser pulses on band-gap materials under the real experimental conditions [4-6]. There is a need to verify the applicability of the Keldysh theory to bulk materials in a wide range of the irradiation parameters and to find ways of determining the photoionization rates beyond its validity limits and beyond the perturbative regime [23].

In this Letter, we first confront the qualitative insights from the Keldysh theory with a Floquet model supported by the density functional theory (DFT). Then, we perform a quantitative comparison of the photoionization rates obtained in the framework of the Keldysh model and in the simulations based on the first-principles time-dependent density functional theory (TDDFT) for a wide range of laser intensities and photon energies for silicon as an example. Finally, based on the TDDFT simulations, we analyze the laser energy absorption and compare it with predictions of the Drude model. Means to improve accuracy of the Drude model for macroscopic description of materials optical response are discussed.

At the basis of the Keldysh photoionization model, there is temporal averaging of a Hamiltonian which describes the interaction of the electromagnetic field with a two-level system, representing an atom, a molecule or a solid in a simplified manner [24]. When performing the temporal averaging of electronic energy levels over one laser cycle [25,26], the so-called laser dressing manifests as a replication of the energy levels spaced by the photon energy. The discrete set of possible quasistates appearing transiently in the energy gap is usually referred as the Wannier-Stark ladder (WSL), the effect which can be described qualitatively using a Floquet model [27].

Figure 1(a) presents a schematics of the WSL quasi-energy levels of silicon shifted by the laser field under the combined action of laser dressing and the optical Stark effect. The ground-state band structure shown on the left was calculated using DFT (see the Supplemental Material [28]). Depending on the choice of laser polarization, wavelength, and intensity, the distances between the quasienergy levels can either increase or decrease in presence of the strong optical field as schematically shown on the right part of Fig. 1(a). Figure 1(b) shows the calculated energies of the laser dressed states as a function of laser intensity, obtained using the Floquet model for the $m=8$ states at the $\Gamma$ point of Si. Note that the dipolar matrix elements [27] were computed using DFT based on the local-density approximation (LDA). With increasing laser intensity, the number of replicas, which are associated with the increasing contribution of the anharmonic motion of the electrons, also increases [26]. With $n=2$ replicas per ground electronic state, the Floquet model reveals multiple crossings (as well as anticrossings, see Ref. [64]) of the dressed electronic levels that may be manifested as a transient metallization during the laser pulse [64]. The latter effect gives birth to important applications in ultrafast optoelectronics $[22,82,83]$.

Let us next consider the results of numerical simulations for silicon irradiated by ultrashort laser pulses, which have been obtained by solving the Kohn-Sham (KS) equations using TDDFT $[11,84]$. The laser field was introduced in the form of a time-dependent vector potential (velocity gauge).
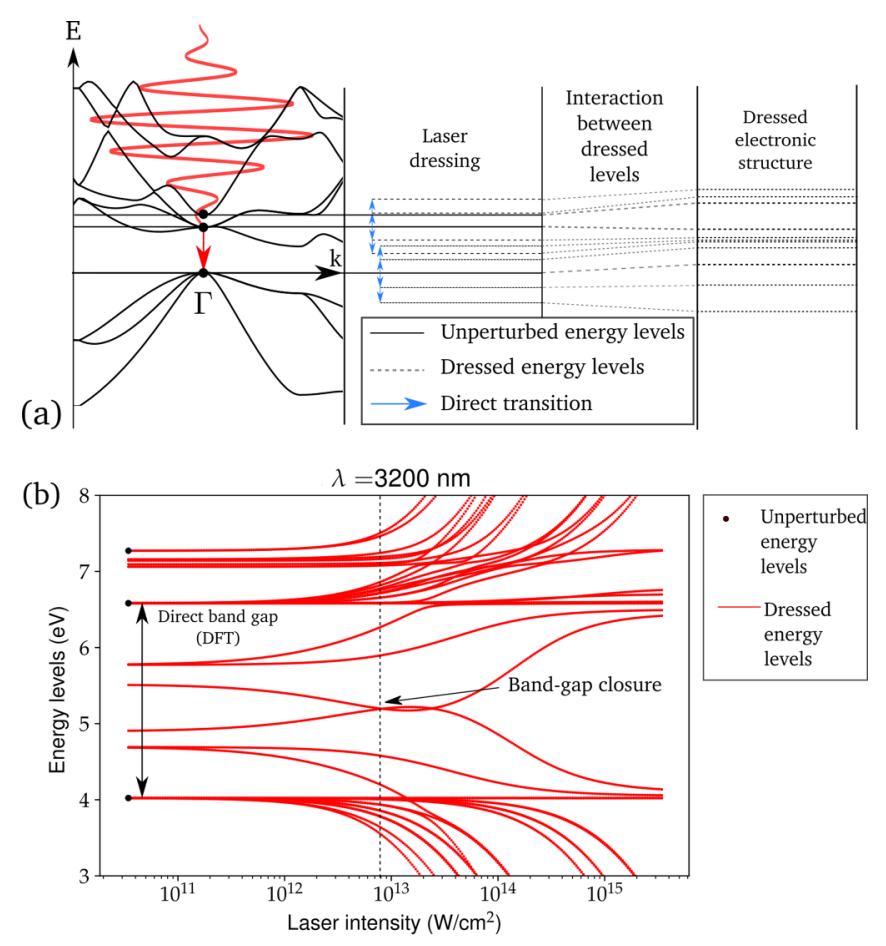

FIG. 1. Schematics (a) and realistic simulation (b) of a transient evolution of band structure in a band-gap solid on the example of a silicon crystal. The electronic bands [(a), left] are dressed by the laser field that results in replica bands at multiples of the driving photon energy [WSL, (a), right]. With increasing laser intensity, a splitting of the electronic levels is observed and the valence and conduction bands experience anticrossing (b) at intensity close to $10^{13} \mathrm{~W} / \mathrm{cm}^{2}$ leading to transient metallization. See further details in the text.

The crystal $\mathrm{Si}<100>$ is modeled by the method of ab initio pseudopotentials [43] (see details in the Supplemental Material [28]) involving periodic boundary conditions. The band structure calculated based on the LDA yields the direct band gap for $\Gamma \rightarrow \Gamma$ transition to be equal to $E_{g}^{\Gamma}=2.56 \mathrm{eV}$ [38]. The approach directly accounts for the effects of the laser field on the valence and conduction electrons from first principles, in particular, at intensities where a perturbative approach is not applicable [85], while also using a more realistic band structure $[12,86,87]$.

Using TDDFT, the number of electrons excited from the valence to the conduction bands, $n_{\text {exc }}$, was calculated using [10]

$$
n_{\mathrm{exc}}(t)=\frac{1}{V}\left[N_{\mathrm{tot}}-\sum_{n, n^{\prime}, \boldsymbol{k}}\left|\int d^{3} \boldsymbol{r} \psi_{n^{\prime}, \boldsymbol{k}}^{*}(\boldsymbol{r}, t) \psi_{n, \boldsymbol{k}}^{\mathrm{GS}}(\boldsymbol{r})\right|^{2}\right],
$$

where $n$ and $n^{\prime}$ are band indices, $\boldsymbol{k}$ indexes the electron wave vectors in three dimensions, $\psi_{n, k}^{\mathrm{GS}}(\boldsymbol{r})$ are the KS wave functions of the ground state, and $\psi_{n^{\prime}, k}^{*}(\boldsymbol{r}, t)$ are the KS time- and space-dependent wave functions, where * indicates complex conjugation. $V$ is the volume of the simulation box (constant in this work), $N_{\text {tot }}$ is the total number of electrons in the simulation volume, expressed by $N_{\mathrm{tot}}=\sum_{n, \boldsymbol{k}}\left|\psi_{n, \boldsymbol{k}}^{\mathrm{GS}}(\boldsymbol{r})\right|^{2}$. The summation is performed over the occupied states in the valence bands. Note that in the ground state, before the laser pulse action, the obtained density of electrons in the conduction bands $n_{\mathrm{exc}}(t=0)$ is zero. The gauge-related problems 

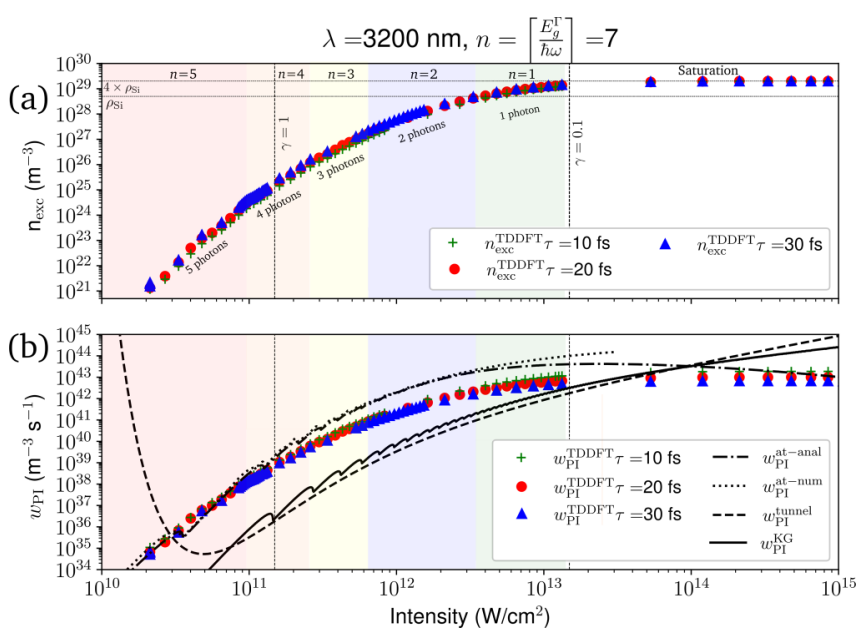

FIG. 2. Density of electrons excited to the conduction bands $n_{\text {exc }}$ by the end of the laser pulse (a) and the excitation rates $w_{\mathrm{PI}}$ (b) as a function of the laser intensity in the bulk of silicon irradiated at $\lambda=3200 \mathrm{~nm}$. By dots, the results of the TDDFT simulations based on the LDA functional are presented for laser pulses of a top-hat temporal shape with durations of 10, 20, and $30 \mathrm{fs}$. In (b), the results of the $\mathrm{KG}$ model ( $w_{\mathrm{PI}}^{\mathrm{KG}}$, black solid line) and the tunneling limit ( $w_{\mathrm{PI}}^{\text {tunnel }}$, black dashed line) are added for comparison. Also for illustration, the data according to the analytical expression for the excitation rate for atoms [1], $w_{\mathrm{PI}}^{\mathrm{at}-\mathrm{anal}}$, and a more exact numerical calculations of integrals of the Keldysh theory $w_{\mathrm{PI}}^{\text {at-num }}$ are shown (see the Supplemental Material [28]).

associated with the virtual excitations were avoided by exploiting the simulation results obtained shortly after the end of the laser irradiation [10]. The KS equation was solved numerically for a number of laser parameters by varying the photon energy $\hbar \omega$ and the laser intensity $I$. Details of the numerical approach are given in the Supplemental Material [28].

Figure 2 presents the results of the TDDFT simulations for 10, 20, and 30 fs laser pulse durations (a "softened" top-hat temporal shape, see details in the Supplemental Material [28]) at the wavelength $\lambda=3200 \mathrm{~nm}(\hbar \omega=0.387 \mathrm{eV})$. The number of photons per electron for crossing the direct band gap can be estimated as $n \sim\left\lceil E_{g}^{\Gamma} / \hbar \omega\right\rceil$, which is seven photons at this wavelength. Using the scaling law $n_{\mathrm{exc}} \sim I^{n}$ [88], the slope of the function $n_{\text {exc }}(I)$ in Fig. 2(a) can be associated with five photons that are needed to cross the band gap at relatively low intensities in the range of $2 \times 10^{10}-1.5 \times 10^{11} \mathrm{~W} / \mathrm{cm}^{2}$ $(\gamma \gtrsim 1)$ [89]. With increasing the laser intensity, the simulations are consistent with experimental trends of a decrease of the $n_{\text {exc }}(I)$ slope [88], which can be conventionally fitted with the $I^{n}$ scaling law at lower $n$ numbers. The corresponding intensity ranges with different $n$ are marked in Fig. 2(a) and shadowed by colors. For each $n$ range of intensity, an effective (pulse averaged) multiphoton ionization coefficient $\sigma_{n}$ (see the Supplemental Material [28]) can be estimated using a simplified formula $\partial n_{\mathrm{exc}} / \partial t \approx n_{\mathrm{exc}}\left(\tau_{p}\right) / \tau_{p}=\sigma_{n} I^{n} / n \hbar \omega$ where $n_{\mathrm{exc}}\left(\tau_{p}\right)$ is the number of the electrons excited to the conduction bands by the end of the laser pulse of duration $\tau_{p}$. For $\tau_{p}=10 \mathrm{fs}$ at $\lambda=3200 \mathrm{~nm}$, the TDDFT results can be fitted with $\sigma_{1}=2.93 \times 10^{6} \mathrm{~m}^{-1}, \sigma_{2}=2 \times 10^{-10} \mathrm{~m} / \mathrm{W}$, $\sigma_{3}=5.25 \times 10^{-26} \mathrm{~m}^{3} \mathrm{~W}^{-2}, \sigma_{4}=3.05 \times 10^{-41} \mathrm{~m}^{5} \mathrm{~W}^{-3}$, and $\sigma_{5}=4.84 \times 10^{-56} \mathrm{~m}^{7} \mathrm{~W}^{-4}$.
Note that in a purely multiphoton regime, the Keldysh theory predicts increasing $n$ with intensity (see Refs. [1,4,17]) while the total photoionization rate can be considered as a sum of $n$-photon processes with different $n$ [14]. Based on ab initio simulations, we interpret the reduction of the photon number $n$ necessary for transition across the band gap as a mutual contribution of laser dressing of electronic states and tunneling that reduces the effective band gap during the laser action. At high intensities when $\gamma<0.1$, the quantity of electrons excited to the conduction bands saturates to the number of electrons available for ionization (four valence electrons per atom in $\mathrm{Si}$ ).

The corresponding excitation rates $w_{\mathrm{PI}}^{\mathrm{TDDFT}}$ are compared with the Keldysh theory in Fig. 2(b). In both approaches, the effective electron mass was taken from the DFT calculations of Ref. [56] ( $m^{*}=0.2226 m_{e}$ with $m_{e}$ to be the electron mass in vacuum). It should be stressed that we use here the Keldysh theory for solids corrected by Gruzdev [8] and referred to further as the KG model [black solid line in Fig. 2(b)]. At laser intensities below $10^{13} \mathrm{~W} / \mathrm{cm}^{2}$, the $w_{\mathrm{PI}}^{\text {TDDFT }}$ values are considerably larger than the KG photoionization rates. This is in line with statements in Refs. [2,8] where it was noticed that the Keldysh theory may underestimate the photoionization rate by order(s) of magnitude. At higher intensities, in the TDDFT simulations the saturation regime is achieved, determined by the number of valence electrons available for ionization. In this regime, the KG rate and its tunneling limit [90] [dashed line in Fig. 2(b)] approach and finally exceed $w_{\mathrm{PI}}^{\mathrm{TD}}$.

The results for the original atomic Keldysh theory, both its analytical expressions and numerical integration, are also added in Fig. 2(b) (dashed-dotted and dotted lines, respectively), obtained for a virtual atom with an effective ionization potential of $2.56 \mathrm{eV}$ [28]. Surprisingly, at intensities below $10^{11} \mathrm{~W} / \mathrm{cm}^{2}$, the atomic theory fits reasonably well the TDDFT results although at higher intensities it deviates from the $a b$ initio data. Important is that replacing the saddle-point approximation in the Keldysh theory by an exact integration has a minor effect, contrary to a conjecture in Ref. [2] (see also the Supplemental Material [28]). As noted in Ref. [91], further advances of the Keldysh theory of photoionization for solids can be seen in introducing the realistic band gap structure as for example proposed by McDonald et al. [54]. However, the rigorous TDDFT simulations presented here represent a solid alternative, both from fundamental and practical points of view. As an illustration, the TDDFT results and their comparisons with the Keldysh theory are given for $\lambda=1600$ and $800 \mathrm{~nm}$ in the Supplemental Material [28].

Comparing $w_{\mathrm{PI}}^{\mathrm{KG}}(I)$ and $w_{\mathrm{PI}}^{\mathrm{TDDFT}}(I)$, one can notice that the $\mathrm{KG}$ model yields oscillations of the multiphoton excitation rate with $I$ while the TDDFT-calculated rates demonstrate rather smooth intensity dependences [Fig. 2(b)]. This difference can also be explained by the two-band approximation applied in the KG model, while TDDFT accounts for multiple bands. We note that the zeros of $n$th order Bessel function correspond to a regular suppression of the effective transition probability called dynamical localization or destruction of tunneling $[66,68]$. In a two-band model, this effect manifests itself as a swift decrease of the excitation rate at certain intensities, whereas in the case of a multiband description, the Bourget theorem secures that only a single transition may 
be simultaneously disabled. As a result, the functions $w_{\mathrm{PI}}(I)$ provided by the TDDFT multiband simulations are smoother than those obtained in the framework of the KG model.

An important comment should be made on the excitation rates derived from the TDDFT simulations and calculated by the Keldysh theory for the saturation regime. In the Keldysh approach, the ionization rate is calculated by averaging the probability over many laser cycles. In our $a b$ initio numerical calculations, electron excitation follows the instantaneous laser field and, hence, it is strongly varying during the period of the electromagnetic wave. Furthermore, valence electrons, which are pushed out to the conduction bands at the first part of the laser pulse, can partially return back to the valence bands when the electric field of the wave changes direction [82,92]. Our estimations of the excitation rates given in Fig. 2(b) for three pulse durations correspond averaging over one, two, and three laser periods (note that one period for $\lambda=3200 \mathrm{~nm}$ is $\sim 10.7 \mathrm{fs}$ ). Thus, in the saturation regime when all or almost all valence electrons are excited to the conduction bands already during the first cycle, the $w_{\mathrm{PI}}^{\mathrm{TDDT}}$ value at $10 \mathrm{fs}$ looks to be more reliable although the excitation rates for all three pulse durations give the same result, transfer of all valence electrons to the conduction bands [Fig. 2(a)]. Note that the KG model does not account for decreasing the number of valence electrons available for excitation into the conduction bands at high intensities and its direct use can lead to an unphysically large number of electrons in the conduction bands [Fig. 2(b)]. When a considerable fraction of valence electrons has been transferred to the conduction bands by the front part of the laser pulse, the excitation rate in the trailing edge of the pulse should naturally be decreasing by the dynamic factor $\left(1-n_{\text {exc }} V / N_{\text {tot }}\right)$ (in our notations; see, e.g., Ref. [93]). Further still, the validity range of the KG theory is limited to single ionization per atom while the TDDFT simulations are not subjected to this limitation.

Analysis of the TDDFT-calculated excitation rates as a function of the photon energy provides insights into the WSL and the Stark shift in bulk crystals. Figure 3 presents the comparison of the excitation rates $w_{\mathrm{PI}}$ calculated by the KG model [Fig. 3(a)] and by TDDFT [Fig. 3(b)] for the $\Gamma \rightarrow \Gamma$ transition in Si. The data are given for the electric field amplitudes $E$ of the electromagnetic wave from 0.5 to $5 \mathrm{~V} / \mathrm{nm}$ (curves from bottom to top). The corresponding intensity range is $3.32 \times 10^{10}-3.32 \times 10^{12} \mathrm{~W} / \mathrm{cm}^{2}$ with the maximum intensity close to the saturation regime for $\lambda=3200 \mathrm{~nm}$ [Fig. 2(a)].

At low electric fields, both the Keldysh formula and the TDDFT simulations reveal regular drops of the electron excitation at the photon energies close to resonances of the band gap at rest (marked by dashed vertical lines at $E_{g}^{\Gamma} / n$ with $n=\{2,3,4,5,6\}$ in Fig. 3). For each $n$ value in Fig. 3(a), the minima at different curves are connected by grey solid lines, indicating the Stark shift effect with increasing laser field strength (compare with inset in the figure where the Stark shift in the KG theory was disregarded). These grey lines are replicated to Fig. 3(b) to guide the eye, showing a similar Stark shift in TDDFT simulations. However, the TDDFT minima sometimes fall beyond the resonant photon energy [e.g., the minima seen between 3 and 4 PA in Fig. 3(b)].

Another qualitative difference between $w_{\mathrm{PI}}^{\mathrm{KG}}(\hbar \omega)$ and $w_{\mathrm{PI}}^{\mathrm{TDDFT}}(\hbar \omega)$ can be admitted for the IR spectral range at high
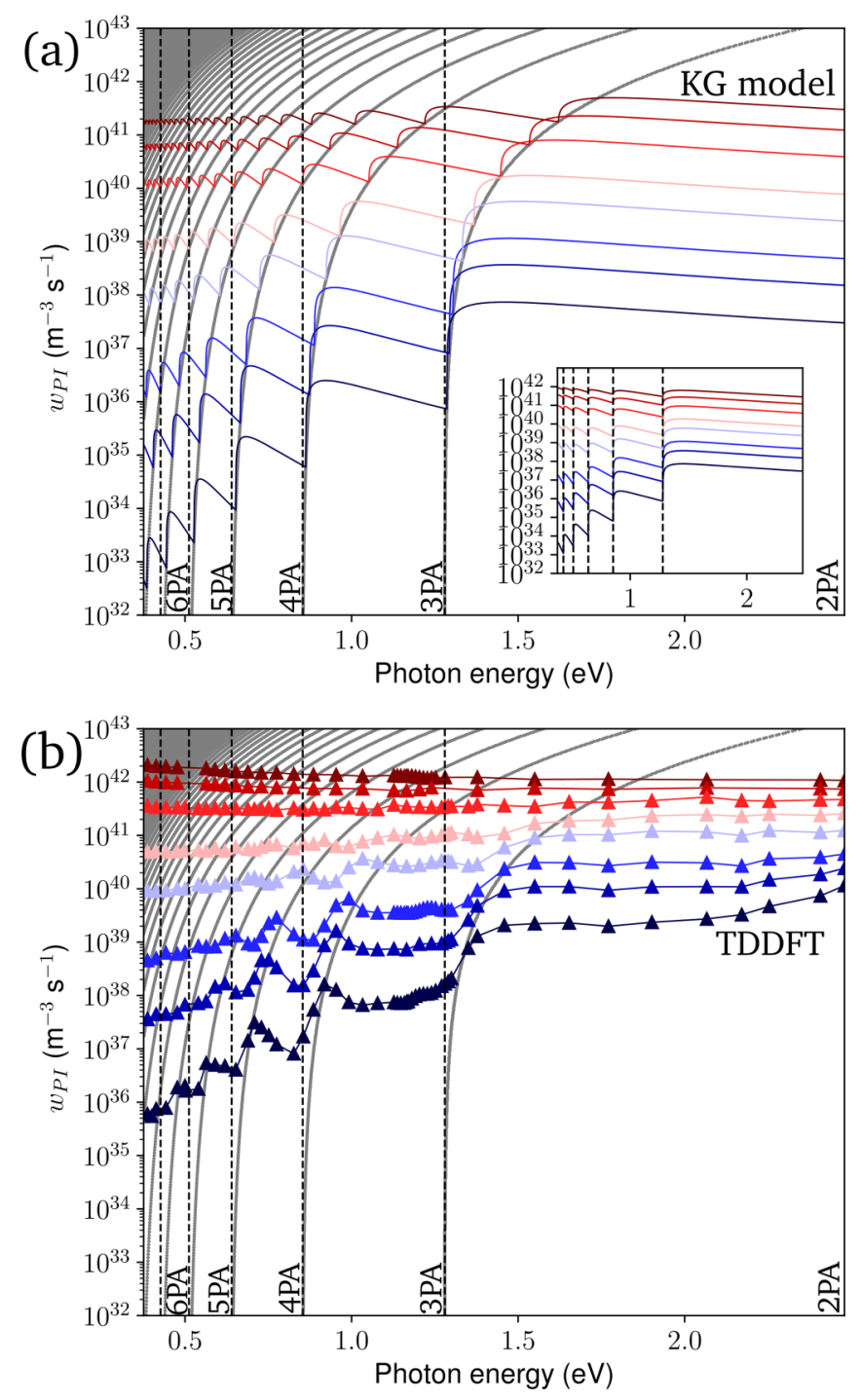

FIG. 3. Excitation rates $w_{\mathrm{PI}}$ as a function of photon energy for $E$ $=0.5,0.75,1.0,1.5,2,3,4,5 \mathrm{~V} / \mathrm{nm}$ (curves from bottom to top), which correspond to $I=0.03,0.08,0.13,0.3,0.53,1.2,2.1,3.3$ $\mathrm{TW} / \mathrm{cm}^{2}$, calculated using (a) the KG model [8] and (b) the TDDFT (LDA functional, band gap of $\mathrm{Si}$ is $2.56 \mathrm{eV}$, pulse duration $\tau=20$ fs). $n$ PA stands for " $n$ photon absorption" where $n$ is an integer. The inset in (a) demonstrates the behavior of the excitation rates when the Stark shift in the KG theory is disregarded.

excitation fields. In the TDDFT simulations the resonance drops are vanishing with increasing laser intensity [Fig. 3(b)]. This is explained by a gradual transition to the tunneling ionization regime. In addition, as noticed above, the KG model does not account for decreasing the number of valence electrons available for excitation into the conduction bands at high intensities that should contribute to vanishing the $w_{\mathrm{PI}}$ resonances. We also note that coupling between moving charges in the valence and conduction bands $[94,95]$ can influence the excitation. The latter effect is not included in the Keldysh theory but can be captured by other approaches [54].

In pump-probe and angle-resolved photoemission experiments $[19,96]$, the possibility to observe the effects of quasienergy levels (the WSL) in bulk band-gap crystals was 


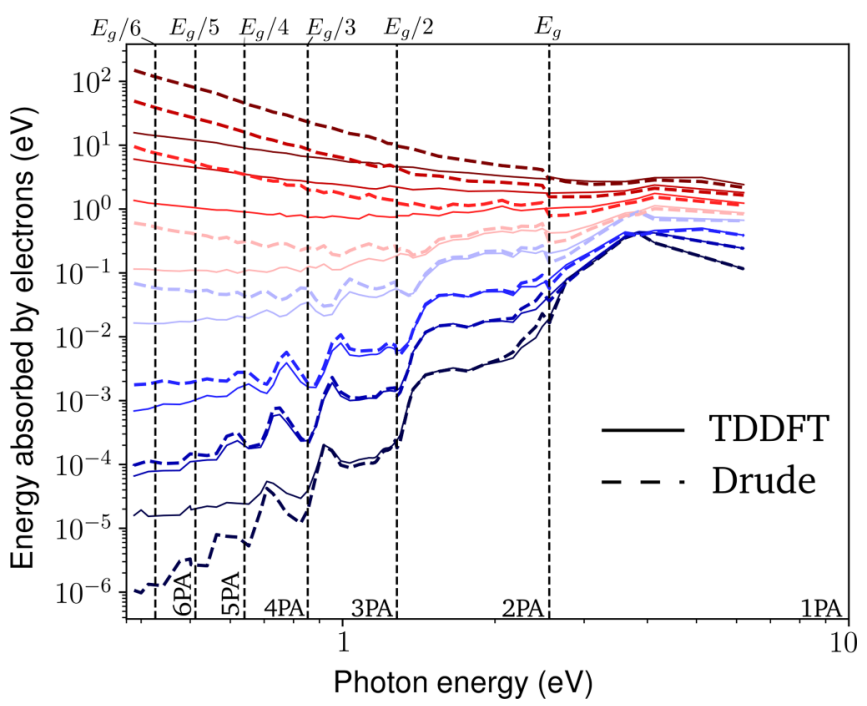

FIG. 4. Energy absorbed by electrons as a function of photon energy for the laser field amplitudes of $E=0.5,0.75,1.0,1.5,2$, $3,4,5 \mathrm{~V} / \mathrm{nm}$ (curves from bottom to top) obtained in the TDDFT simulations (solid lines), and using a macroscopic Drude model for the densities of the excited electrons calculated by the TDDFT (dashed lines). The energy is given per one atom of silicon crystal. All calculations were performed for the top-hat temporal shape of laser pulses with duration of $20 \mathrm{fs}$.

recently proven. Here, based on the TDDFT simulations of the laser energy absorbed by electrons (see solid lines in Fig. 4 and the Supplemental Material [28]), we anticipate a possibility to directly observe the WSL by measuring the change in time-integrated optical transmission when accurately tuning the pump laser wavelength, preferably in the IR spectral range. Another fundamental aspect, which is of high importance for multiphysics large-scale modeling of the interaction of ultrashort laser pulses with band-gap materials, is an adequate description of dynamically changing reflectivity and absorptivity, which are usually treated in the framework of the Drude model [97]. The TDDFT simulations give an excellent opportunity to verify on how accurate can be this simplest optical theory based on the classical equations of electron motion in an optical electric field.

TDDFT and the Drude model have been compared based on calculations of the laser energy absorption at a wide range of laser parameters (Fig. 4). For this aim, the TDDFTcalculated density of the laser-excited electrons $n_{\mathrm{exc}}^{\mathrm{TDDT}}$ was introduced into the Drude formalism coupled with the energy balance equation for the electrons absorbing laser radiation. The latter was integrated for 20-fs laser pulses of the same shape as in the TDDFT simulations (see details in the Supplemental Material [28]). An extensive series of simulations was performed with varying the Drude damping time $\tau_{D}$ for finding the best fit to the TDDFT results. By employing $\tau_{D}=$ 6 fs for all tested wavelengths and field strengths, it is possible to obtain a reasonable agreement of the Drude modeling data with the TDDFT results (Fig. 4). A discrepancy between them becomes pronounced for longer wavelengths and stronger optical fields. This can be attributed to the fact that $\tau_{D}$ is dependent on the density of electrons, their energy, and laser wavelength [97,98]. Thus, the TDDFT simulations can provide a possibility to derive the $\tau_{D}$ values for a wide range of laser irradiation conditions and for different materials.

In conclusion, based on the TDDFT simulations we have investigated photoionization of crystalline silicon by ultrashort laser pulses in a wide range of laser intensities and for photon energies from UV to mid-IR spectral range. The excitation rates have been derived and compared with the Keldysh theory [1]. The photoionization rates obtained within the Keldysh approach in its validity range are smaller by more than an order of magnitude as compared with the TDDFT simulation results that is in agreement with previous observations $[2,8]$ that can be attributed to several factors, including simplification of the band structure [91]. We anticipate that TDDFT predicts well the photoionization process both within and beyond the limits of applicability of the Keldysh formalism. The excitation rates obtained in the firstprinciples simulations represent valuable fundamental and practical information and, being tabulated in a wide range of the irradiation parameters, can be directly used in multiphysics large-scale models, such as laser beam propagation in band-gap materials [99-101].

By calculating the nonlinear absorption as a function of photon energy for the Si crystal, we observed the quasi-energy levels known as the Wannier-Stark ladder. The energy of these laser-dressed states are shifted by the Stark effect at high optical field strengths. With further increasing field strength, these levels become less pronounced and finally disappear in the TDDFT simulation results. Finally, we have verified the Drude model based on laser energy absorption calculated in the TDDFT framework and a simplified energy balance equation, thus showing that the TDDFT simulations can potentially provide reliable data on the electron damping time as a function of density and energy of electrons and laser wavelength.

It should be emphasized that the results of the present work refer to pure photoionization-related processes in spatially homogeneous optical fields (dipolar approximation) which are not masked by electron-phonon coupling. For longer pulse durations than used here, the role of phonons becomes important, leading to indirect transitions, which are not accounted for in the present simulations. Furthermore, at longer timescales, lattice destabilization, nonthermal melting, Auger recombination, transport of hot quasifree charge carries and electron energy relaxation are important for silicon and other semiconductors $[92,97,98,102,103]$, which are beyond the scope of this paper.

The research of T.J.-Y.D., V.P.Z., and N.M.B. is financed by the European Regional Development Fund and the state budget of the Czech Republic (Project No. BIATRI: CZ.02.1.01/0.0/0.0/15_003/0000445, Project HiLASE CoE: No. CZ.02.1.01/0.0/0.0/15_006/0000674). T.J.-Y.D. also acknowledges funding from the European Union's Horizon 2020 research and innovation programme under the Marie Skłodowska-Curie Actions Individual Fellowship Grant agreement No. 657424 and staff exchange Grant agreement No. 823897. This work was partially supported by the Ministry of Education, Youth and Sports of the Czech 
Republic through the e-INFRA CZ (ID:90140). N.T.-D., H.A. and A.R. acknowledge financial support from the European Research Council (ERC-2015-AdG694097), the Cluster of Excellence Advanced Imaging of Matter (AIM), Grupos Consolidados (IT1249-19) and Deutsche Forschungsgemeinschaft (DFG) SFB-925 Project 170620586. Computational resources for data archiving were supplied by the project “e-Infrastruktura CZ" (e-INFRA CZ LM2018140) supported by the Ministry of Education, Youth and Sports of the Czech Republic. The DECI resource "Prometheus" based in Poland at Krakow with support from the PRACE aisbl (Project BOLERO) is greatly appreciated.
[1] L. V. Keldysh, J. Exptl. Theoret. Phys. (U.S.S.R.) 47, 1945 (1964) [Sov. Phys. JETP 20, 1307 (1965)].

[2] A. Vaidyanathan, T. W. Walker, A. H. Guenther, S. S. Mitra, and L. M. Narducci, Phys. Rev. B 20, 3526 (1979).

[3] H. Deng, W. Guo, H. Gao, L. Li, X. Yuan, W. Zheng, and X. Zu, J. Opt. 21, 075501 (2019).

[4] A. Kaiser, B. Rethfeld, M. Vicanek, and G. Simon, Phys. Rev. B 61, 11437 (2000).

[5] B. Chimier, O. Utéza, N. Sanner, M. Sentis, T. Itina, P. Lassonde, F. Légaré, F. Vidal, and J. C. Kieffer, Phys. Rev. B 84, 094104 (2011).

[6] L. Gallais, D.-B. Douti, M. Commandré, G. Batavičiuté, E. Pupka, M. Ščiuka, L. Smalakys, V. Sirutkaitis, and A. Melninkaitis, J. Appl. Phys. 117, 223103 (2015).

[7] M. Lenzner, J. Krüger, S. Sartania, Z. Cheng, C. Spielmann, G. Mourou, W. Kautek, and F. Krausz, Phys. Rev. Lett. 80, 4076 (1998).

[8] V. E. Gruzdev, Opt. Eng. 53, 122515 (2014).

[9] S. V. Popruzhenko, J. Phys. B 47, 204001 (2014).

[10] T. Otobe, M. Yamagiwa, J.-I. Iwata, K. Yabana, T. Nakatsukasa, and G. F. Bertsch, Phys. Rev. B 77, 165104 (2008).

[11] K. Yabana, T. Sugiyama, Y. Shinohara, T. Otobe, and G. F. Bertsch, Phys. Rev. B 85, 045134 (2012).

[12] A. Yamada and K. Yabana, Eur. Phys. J. D 73, 87 (2019).

[13] N. S. Shcheblanov, M. E. Povarnitsyn, P. N. Terekhin, S. Guizard, and A. Couairon, Phys. Rev. A 96, 063410 (2017).

[14] T. Otobe, Y. Shinohara, S. A. Sato, and K. Yabana, J. Phys. Soc. Jpn. 88, 024706 (2019).

[15] A. N. Pfeiffer and S. R. Leone, Phys. Rev. A 85, 053422 (2012).

[16] M. Anand, S. Pabst, O. Kwon, and D. E. Kim, Phys. Rev. A 95, 053420 (2017).

[17] J. R. Gulley, Opt. Eng. 51, 121805 (2012).

[18] E. J. Sie, J. W. McIver, Y.-H. Lee, L. Fu, J. Kong, and N. Gedik, Nat. Mater. 14, 290 (2014).

[19] C. Schmidt, J. Bühler, A.-C. Heinrich, J. Allerbeck, R. Podzimski, D. Berghoff, T. Meier, W. G. Schmidt, C. Reichl, W. Wegscheider, D. Brida, and A. Leitenstorfer, Nat. Commun. 9, 2890 (2018).

[20] M. Schultze, K. Ramasesha, C. D. Pemmaraju, S. A. Sato, D. Whitmore, A. Gandman, J. S. Prell, L. J. Borja, D. Prendergast, K. Yabana et al., Science 346, 1348 (2014).

[21] T. Winkler, L. Haahr-Lillevang, C. Sarpe, B. Zielinski, N. Götte, A. Senftleben, P. Balling, and T. Baumert, Nat. Phys. 14, 74 (2017).

[22] O. Kwon, T. Paasch-Colberg, V. Apalkov, B.-K. Kim, J.-J. Kim, M. I. Stockman, and D. Kim, Sci. Rep. 6, 21272 (2016).

[23] M. Uemoto, Y. Kuwabara, S. A. Sato, and K. Yabana, J. Chem. Phys. 150, 094101 (2019).
[24] L. V. Keldysh, J. Exptl. Theoret. Phys. (U.S.S.R.) 33, 994 (1957) [Sov. Phys. JETP 6, 763 (1958)].

[25] T. Higuchi, M. I. Stockman, and P. Hommelhoff, Phys. Rev. Lett. 113, 213901 (2014).

[26] U. De Giovannini, H. Hübener, and A. Rubio, Nano Lett. 16, 7993 (2016).

[27] The time-averaged Hamiltonian $H$ is expressed as $[25,26$, and Supplemental Material in Ref. [26]] $H=\frac{\omega}{2 \pi} \int_{0}^{\frac{2 \pi}{\omega}} d t^{\prime}$ $\exp [i(m-n) \omega t] H_{F}\left(t^{\prime}\right)+\delta_{m, n} m \omega$ where the Floquet Hamiltonian $H_{F}(t)$, as an example of a two-level system with the band gap energy $E_{g}$ at $\Gamma$-point, is given by $H_{F}(t)=$ $\left[\begin{array}{cc}E_{g} / 2 & 0 \\ 0 & -E_{g} / 2\end{array}\right]+A \cos (\omega t)\left[\begin{array}{cc}0 & M_{12} \\ M_{21} & 0\end{array}\right]$. Here, $M_{12}$ and $M_{21}$ are the material-dependent dipolar matrix elements characterizing the dipolar transitions between two electronic levels (indexed by 1 and 2); $A$ is the field strength; $\omega$ is the photon frequency. Note that this model can easily be extended to $n$ bands exchanging $m$ photons [25].

[28] See Supplemental Material at http://link.aps.org/ supplemental/10.1103/PhysRevB.104.L241201 for details on the models employed in this study with referencing to [1,4,8,10,11,19,22,26,29-81], additional TDDFT results, the excitation rates obtained from TDDFT for different wavelengths and laser intensity ranges, and an URL of our database of the excitation rates.

[29] E. K. Gross, J. F. Dobson, and M. Petersilka, in Density Functional Theory II (Springer, Berlin, 1996), pp. 81-172.

[30] M. A. L. Marques, N. T. Maitra, F. M. S. Nogueira, E. K. U. Gross, and A. Rubio, eds., Fundamentals of TimeDependent Density Functional Theory, Lecture Notes in Physics (Springer, New York, 2011).

[31] M. Ruggenthaler, N. Tancogne-Dejean, J. Flick, H. Appel, and A. Rubio, Nat. Rev. Chem. 2, 0118 (2018).

[32] C. A. Ullrich, Time-Dependent Density-Functional Theory: Concepts and Applications (Oxford University Press, New York, 2012).

[33] F. Tran and P. Blaha, Phys. Rev. Lett. 102, 226401 (2009).

[34] E. K. U. Gross and W. Kohn, Phys. Rev. Lett. 55, 2850 (1985).

[35] R. W. Godby, M. Schlüter, and L. J. Sham, Phys. Rev. Lett. 56, 2415 (1986).

[36] O. Sugino and R. Car, Phys. Rev. Lett. 74, 1823 (1995).

[37] J. M. Zuo, P. Blaha, and K. Schwarz, J. Phys.: Condens. Matter 9, 7541 (1997).

[38] D. Waroquiers, A. Lherbier, A. Miglio, M. Stankovski, S. Poncé, M. J. T. Oliveira, M. Giantomassi, G.-M. Rignanese, and X. Gonze, Phys. Rev. B 87, 075121 (2013).

[39] S. A. Sato, Y. Taniguchi, Y. Shinohara, and K. Yabana, J. Chem. Phys. 143, 224116 (2015).

[40] M. A. Marques, A. Castro, G. F. Bertsch, and A. Rubio, Comput. Phys. Commun. 151, 60 (2003). 
[41] A. Castro, H. Appel, M. Oliveira, C. A. Rozzi, X. Andrade, F. Lorenzen, M. A. L. Marques, E. K. U. Gross, and A. Rubio, Phys. Status Solidi B 243, 2465 (2006).

[42] X. Andrade, J. Alberdi-Rodriguez, D. A. Strubbe, M. J. T. Oliveira, F. Nogueira, A. Castro, J. Muguerza, A. Arruabarrena, S. G. Louie, A. Aspuru-Guzik, A. Rubio, and M. A. L. Marques, J. Phys.: Condens. Matter 24, 233202 (2012).

[43] N. Troullier and J. L. Martins, Phys. Rev. B 43, 1993 (1991).

[44] A. Castro, M. A. L. Marques, and A. Rubio, J. Chem. Phys. 121, 3425 (2004).

[45] C. Mézel, A. Bourgeade, and L. Hallo, Phys. Plasmas 17, 113504 (2010).

[46] J. R. Gulley and W. M. Dennis, Phys. Rev. A 81, 033818 (2010).

[47] J. R. Gulley, S. W. Winkler, W. M. Dennis, C. M. Liebig, and R. Stoian, Phys. Rev. A 85, 013808 (2012).

[48] J. R. Gulley and T. E. Lanier, Phys. Rev. B 90, 155119 (2014).

[49] J. R. Gulley, J. Liao, and T. E. Lanier, Proc. SPIE 8972, 89720T (2014).

[50] V. E. Gruzdev, Phys. Rev. B 75, 205106 (2007).

[51] V. E. Gruzdev, AIP Conf. Proc. 1278, 131 (2010).

[52] P. Jürgens, M. Jupé, M. Gyamfi, and D. Ristau, Proc. SPIE 10014, 100141C (2016).

[53] C. R. McDonald, G. Vampa, G. Orlando, P. B. Corkum, and T. Brabec, J. Phys.: Conf. Ser. 594, 012021 (2015).

[54] C. R. McDonald, G. Vampa, P. B. Corkum, and T. Brabec, Phys. Rev. Lett. 118, 173601 (2017).

[55] V. Turkowski and C. A. Ullrich, Phys. Rev. B 77, 075204 (2008).

[56] J. Laflamme Janssen, Y. Gillet, S. Poncé, A. Martin, M. Torrent, and X. Gonze, Phys. Rev. B 93, 205147 (2016).

[57] L. N. Gaier, M. Lein, M. I. Stockman, P. L. Knight, P. B. Corkum, M. Y. Ivanov, and G. L. Yudin, J. Phys. B 37, L57 (2004).

[58] M. Partanen, T. Häyrynen, J. Oksanen, and J. Tulkki, Phys. Rev. A 95, 063850 (2017).

[59] L. Fritsche, Phys. Status Solidi B 13, 487 (1966).

[60] C. Cohen-Tannoudji and S. Reynaud, J. Phys. B 10, 345 (1977).

[61] J. Flick, D. M. Welakuh, M. Ruggenthaler, H. Appel, and A. Rubio, ACS Photonics 6, 2757 (2019).

[62] V. Rokaj, M. Ruggenthaler, F. G. Eich, and A. Rubio, arXiv:2006.09236 (2021).

[63] M. Durach, A. Rusina, M. F. Kling, and M. I. Stockman, Phys. Rev. Lett. 105, 086803 (2010).

[64] M. Durach, A. Rusina, M. F. Kling, and M. I. Stockman, Phys. Rev. Lett. 107, 086602 (2011).

[65] V. Rokaj, D. M. Welakuh, M. Ruggenthaler, and A. Rubio, J. Phys. B 51, 034005 (2018).

[66] T. Tamaya and T. Kato, Phys. Rev. B 100, 081203(R) (2019).

[67] T. Kato, K. Nobusada, and S. Saito, J. Phys. Soc. Jpn. 89, 024301 (2020).

[68] P. Xia, T. Tamaya, C. Kim, F. Lu, T. Kanai, N. Ishii, J. Itatani, H. Akiyama, and T. Kato, Phys. Rev. B 104, L121202 (2021).

[69] G. H. Wannier, Phys. Rev. 117, 432 (1960).

[70] G. H. Wannier, Phys. Rev. 181, 1364 (1969).

[71] G. N. Watson, A Treatise on the Theory of Bessel Functions (Cambridge University Press, Cambridge, 1995).
[72] R. M. Martin, Electronic Structure: Basic Theory and Practical Methods (Cambridge University Press, Cambridge, 2004).

[73] B. C. Stuart, M. D. Feit, S. Herman, A. M. Rubenchik, B. W. Shore, and M. D. Perry, Phys. Rev. B 53, 1749 (1996).

[74] S. Pearl, N. Rotenberg, and H. M. van Driel, Appl. Phys. Lett. 93, 131102 (2008).

[75] A. D. Bristow, N. Rotenberg, and H. M. van Driel, Appl. Phys. Lett. 90, 191104 (2007).

[76] D. Reitze, T. Zhang, W. M. Wood, and M. C. Downer, J. Opt. Soc. Am. B 7, 84 (1990).

[77] T. Sjodin, H. Petek, and H.-L. Dai, Phys. Rev. Lett. 81, 5664 (1998).

[78] T. F. Boggess, S. C. Moss, I. W. Boyd, and A. L. Smirl, Opt. Lett. 9, 291 (1984).

[79] E. D. Palik, Handbook of Optical Constants of Solids (Academic Press, New York, 1985).

[80] J. Shah, R. F. Leheny, and C. Lin, Solid State Commun. 18, 1035 (1976).

[81] M. Noda, K. Iida, M. Yamaguchi, T. Yatsui, and K. Nobusada, Phys. Rev. Appl. 11, 044053 (2019).

[82] A. Schiffrin, T. Paasch-Colberg, N. Karpowicz, V. Apalkov, D. Gerster, S. Mühlbrandt, M. Korbman, J. Reichert, M. Schultze, S. Holzner, J. V. Barth, R. Kienberger, R. Ernstorfer, V. S. Yakovlev, M. I. Stockman, and F. Krausz, Nature (London) 493, 70 (2012).

[83] M. Schultze, E. M. Bothschafter, A. Sommer, S. Holzner, W. Schweinberger, M. Fiess, M. Hofstetter, R. Kienberger, V. Apalkov, V. S. Yakovlev, M. I. Stockman, and F. Krausz, Nature (London) 493, 75 (2012).

[84] X. Andrade, D. Strubbe, U. De Giovannini, A. H. Larsen, M. J. T. Oliveira, J. Alberdi-Rodriguez, A. Varas, I. Theophilou, N. Helbig, M. J. Verstraete, L. Stella, F. Nogueira, A. Aspuru-Guzik, A. Castro, M. A. L. Marques, and A. Rubio, Phys. Chem. Chem. Phys. 17, 31371 (2015).

[85] Applicability of perturbative nonlinear optics is believed to be valid for relatively small optical driver fields as compared to a characteristic atomic field strength $E_{\text {at }}$. [104]. In Si, the $E_{\text {at }}$ value can be estimated as $\sim 26 \mathrm{~V} / \mathrm{nm}$ [93]. We note that, according to recent studies [105], optical nonlinearities related to photoionization become nonperturbative at even smaller fields, which are defined by the Keldysh parameter $\gamma$.

[86] M. Lucchini, S. A. Sato, A. Ludwig, J. Herrmann, M. Volkov, L. Kasmi, Y. Shinohara, K. Yabana, L. Gallmann, and U. Keller, Science 353, 916 (2016).

[87] T. Otobe, Y. Shinohara, S. A. Sato, and K. Yabana, Phys. Rev. B 93, 045124 (2016).

[88] V. V. Temnov, K. Sokolowski-Tinten, P. Zhou, A. ElKhamhawy, and D. von der Linde, Phys. Rev. Lett. 97, 237403 (2006).

[89] For $1600 \mathrm{~nm}$ and $800 \mathrm{~nm}$ wavelengths (see Ref. [28]), the slope of the $n_{\text {exc }}(I)$, obtained in the TDDFT simulations at low intensities, corresponds to $n=\left\lceil E_{\mathrm{g}}^{\Gamma} / \hbar \omega\right\rceil$.

[90] The employed formula is available in Ref. [4] and in the Supplemental Material [28].

[91] S. M. Golin, S. E. Kirkwood, D. D. Klug, D. M. Villeneuve, D. M. Rayner, C. A. Trallero Herrero, and P. B. Corkum, J. Phys. B 47, 204025 (2014).

[92] S. A. Sato, K. Yabana, Y. Shinohara, T. Otobe, and G. F. Bertsch, Phys. Rev. B 89, 064304 (2014). 
[93] N. M. Bulgakova, R. Stoian, A. Rosenfeld, I. V. Hertel, W. Marine, and E. E. B. Campbell, Appl. Phys. A 81, 345 (2005).

[94] T. Higuchi, C. Heide, K. Ullmann, H. B. Weber, and P. Hommelhoff, Nature (London) 550, 224 (2017).

[95] F. Schlaepfer, M. Lucchini, S. A. Sato, M. Volkov, L. Kasmi, N. Hartmann, A. Rubio, L. Gallmann, and U. Keller, Nat. Phys. 14, 560 (2018).

[96] F. Mahmood, C.-K. Chan, Z. Alpichshev, D. Gardner, Y. Lee, P. A. Lee, and N. Gedik, Nat. Phys. 12, 306 (2016).

[97] K. Sokolowski-Tinten and D. von der Linde, Phys. Rev. B 61, 2643 (2000).

[98] S. A. Sato, Y. Shinohara, T. Otobe, and K. Yabana, Phys. Rev. B 90, 174303 (2014).
[99] L. Sudrie, A. Couairon, M. Franco, B. Lamouroux, B. Prade, S. Tzortzakis, and A. Mysyrowicz, Phys. Rev. Lett. 89, 186601 (2002).

[100] N. M. Bulgakova, V. P. Zhukov, and Y. P. Meshcheryakov, Appl. Phys. B 113, 437 (2013).

[101] V. P. Zhukov, S. Akturk, and N. M. Bulgakova, J. Opt. Soc. Am. B 36, 1556 (2019).

[102] C. V. Shank, R. Yen, and C. Hirlimann, Phys. Rev. Lett. 50, 454 (1983).

[103] T. J.-Y. Derrien, T. E. Itina, R. Torres, T. Sarnet, and M. Sentis, J. Appl. Phys. 114, 083104 (2013).

[104] R. W. Boyd, Nonlinear Optics (Academic Press, New York, 2008).

[105] A. Zheltikov, Opt. Express 27, 8246 (2019). 